\title{
In vitro transcribed sgRNA causes cell death by inducing interferon release
}

\section{Dear editor}

The clustered regularly interspaced short palindromic repeats (CRISPR) and the CRISPR-associated proteins 9 (Cas9) systems are powerful tools for gene editing. Ribonucleoprotein (RNP) complex composed of Cas9 protein and sgRNA binds to specific genomic loci and generate DNA double strand breaks. While plasmids expressing Cas9 protein and sgRNA are routinely transfected into various cell lines to perform gene editing (Cong et al., 2013; Mali et al., 2013; Ran et al., 2013), direct delivery of Cas9-sgRNA RNP has shown higher efficiency and lower off-target effects (Kim et al., 2014), especially in human primary cells such as T cells (Hendel et al., 2015; Schumann et al., 2015). SgRNA can be generated by either in vitro transcription (IVT) or chemical synthesis. IVT is widely used to generate sgRNAs, since it can be easily performed in most labs.

Gene editing of human primary cells, such as human CD34 ${ }^{+}$hematopoietic stem and progenitor cells (HSPCs), T cells and chimeric antigen receptor $T$ (CAR-T) cells, is important for studying gene functions in these cell types, and holds great promise to further improve cell therapy (Schumann et al., 2015; Dever et al., 2016; Liu et al., 2016; Ren et al., 2017; Zhang et al., 2017). Our previous study showed that multiplex gene editing using CRISPR-Cas9 RNP hampered the proliferation of CAR-T cells (Liu et al., 2016). It was also reported that Cas9/hCD45sg1 RNP-treated human HSPCs had lower cell number compared to Cas9 proteintreated control (Gundry et al., 2016). In this study, we investigated the mechanism of cell loss after electroporation of Cas9-sgRNA RNP and find an easy method to resolve it.

As our initial attempt of gene editing in human CD34 ${ }^{+}$ HSPCs, we delivered Cas9-sgRNA RNPs into HSPCs by electroporation, using sgRNA in vitro transcribed by $\mathrm{T} 7$ polymerase. We observed significantly decreased cell number and reduced CD34 expression in survived cells $48 \mathrm{~h}$ after electroporation in experiments using five different sgRNA (Fig. S1A and S1B). Accordingly, the colony forming ability of HSPCs after RNP electroporation was markedly compromised (Fig. S1C). To identify the factor that contributed to this effect, we delivered IVT sgRNA (sgRNA-IVT), RNP complex consisting of Cas9 protein and IVT sgRNA (RNP-IVT), or Cas9 protein alone individually into primary
HSPCs by electroporation. We observed lower cell viability, reduced CD34 expression and decreased colony forming ability in sgRNA-IVT and RNP-IVT groups, while the cells in Cas9 protein group and mock electroporation group survived well (Fig. 1A-C), indicating that sgRNA-IVT reduced HSPC stemness in addition to causing cell death.

To validate whether sgRNA could also cause the death of T cells, we electroporated different amounts of IVT sgRNA into human primary $\mathrm{CD}^{+} \mathrm{T}$ cells. Reduced cell viability and increased immune stimulation were observed when higher amounts of sgRNAs were used (Fig. S2A).

All sgRNAs we tested had a negative effect on the survival of HSPCs and T cells, suggesting that some common feature of these IVT sgRNA was responsible for this effect. Based on existing reports, we hypothesized that the 5 , triphosphate of IVT sgRNA induced type I IFN production, leading to cell death in HSPCs and T cells. To test this hypothesis, we electroporated sgRNA-IVT targeting different genomic loci into $\mathrm{CD} 34^{+} \mathrm{HSPCs}$ and $\mathrm{CD}^{+}{ }^{+} \mathrm{T}$ cells respectively, and measured the concentration of type I IFN in the culture medium using enzyme-linked immunosorbent assay (ELISA). Indeed, we detected significant release of IFN I in all samples electroporated with RNP-IVT (Figs. 1D and S2B).

Next we explored the effects of type I IFN on human primary cells, we cultured freshly isolated HSPCs or $\mathrm{CD}^{+} \mathrm{T}$ cells with IFN I at different concentration. We found that the survival and CD34 expression of HSPC were decreased drastically along with the increased concentration of IFN- $\alpha$. However, IFN- $\beta$ had no obvious effect (Fig. 1E and 1F). Similar results were obtained in $\mathrm{CD}^{+} \mathrm{T}$ cells (Fig. S3A). Using Caspase-Glo ${ }^{R}$ 3/7 assay, we detected significantly increased caspase activity in $T$ cells cultured with IFN- $\alpha$ (Fig. S3B). These data confirmed that IVT sgRNA induced IFN- $\alpha$ release, which led to the apoptosis of primary cells.

To verify whether the 5' triphosphate of sgRNA induced the production of IFN I, we removed the 5' triphosphates of sgRNA using calf intestine phosphatase (CIP) (Dong-Ho et al., 2004) and chemically synthesized a 5'-hydroxylated (5'-OH) sgRNA targeting AAVS1 site. The viability of HSPCs and $T$ cells electroporated with CIP-treated and chemically synthesized sgRNA together with Cas9 protein were similar to control, which was significantly higher than RNP-IVT 


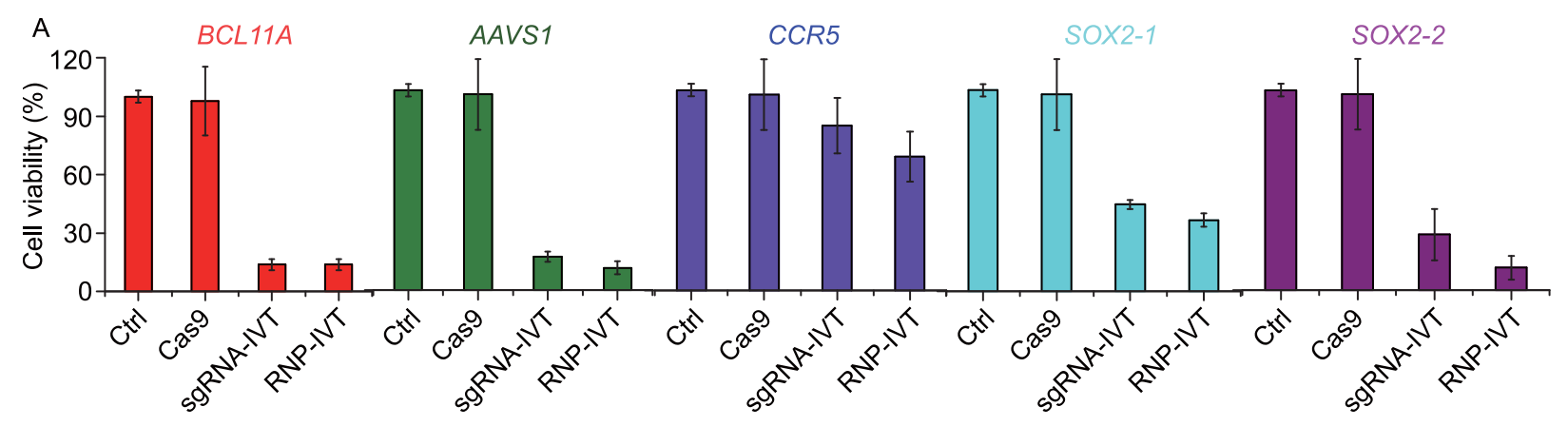

B BCL11A

AAVS1

CCR5

sox2-2
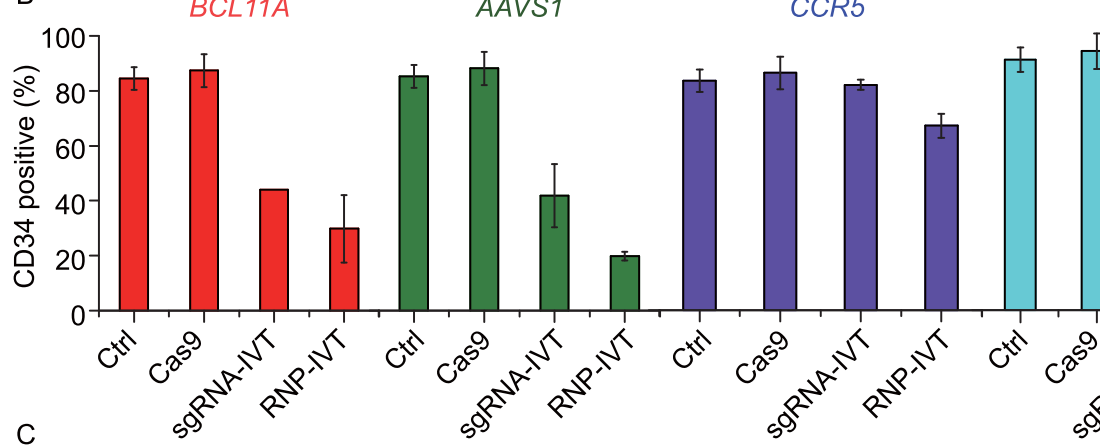

SOX2-1

SOX2-2
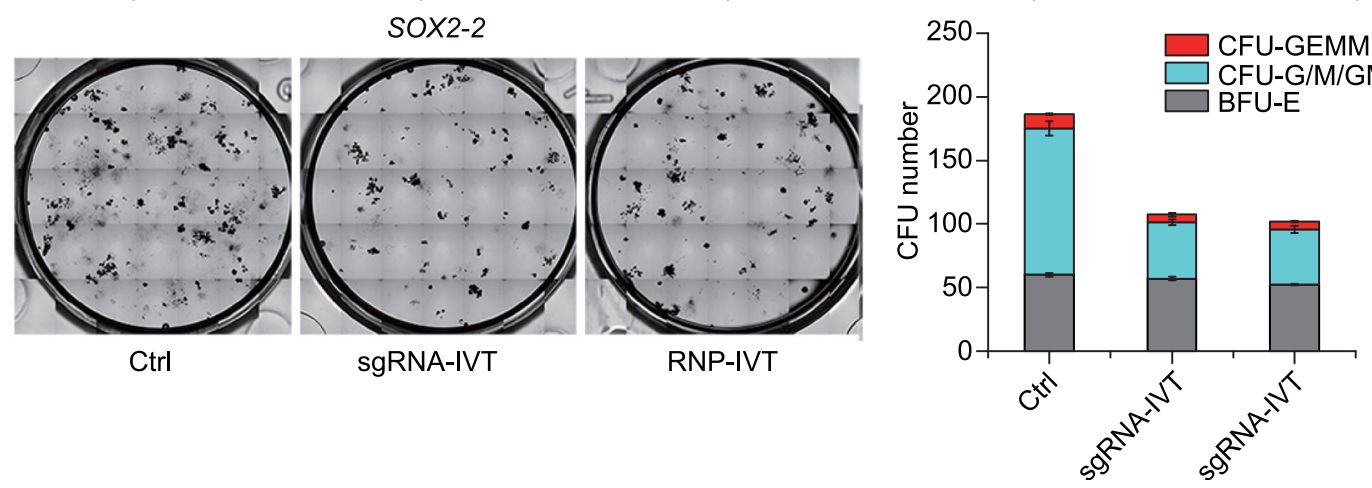

Figure 1. IVT sgRNA induced the production of IFN- $\alpha$, which lead to severe cell death and stemness reduction in human CD34 ${ }^{+}$HSPCs. (A-C) Cell viability (A), CD34 expression (B) and colony forming units (C) of human primary HSPCs electroporated with indicated samples. Cell viabilities and CD34 expression were measured $48 \mathrm{~h}$ post electroporation by cell counting and FACS respectively. For colony forming unit (CFU) assay, same number of viable cells were seeded $48 \mathrm{~h}$ post electroporation, the colony number and lineage differentiation were counted and analyzed two weeks later. In (A), (B), (C) experiments were performed in HSPCs from two donors. Error bars represent SD for two biological replicates. (D) Type I IFN production in HSPCs after electroporation. RNPs targeting indicated sites were delivered into HSPCs. The IFN concentration in medium supernatant were measured $24 \mathrm{~h}$ post electroporation by ELISA, respectively (mean $\pm \mathrm{SD}, n=3$ ). (E and F) The cell viability (mean $\pm \mathrm{SD}, n=3$ ) (E) and CD34 expression $(\mathrm{F})$ of HSPCs after co-culture with IFN in different concentrations.

samples (Fig. S4A and S4B). Consistently, the caspase activity induced by sgRNA without 5 ' triphosphate was much lower compared with sgRNA containing 5' triphosphate (Fig. S4C and S4D). These results demonstrated that the 5' triphosphate of sgRNA is the major factor that induced innate immunity and primary cell death.

To confirm this finding at multiple genomic loci, we electroporated IVT sgRNA with CIP treatment alone or in RNP complex, targeting BCL11A and other four sites, into HSPCs.
Indeed, CIP-treated sgRNA (sgRNA-CIP) did not induce IFN release (Figs. $2 \mathrm{~A}$ and $\mathrm{S} 5 \mathrm{~A}$ ), and led to significantly improved survival rate and colony formation capability of HSPCs, to a level comparable to the mock electroporation control (Figs. 2B, 2C, S5B and S5C). To detect the stemness of electroporated HSPCs more comprehensively, we further analyzed the colony formation capability of granulocyte, erythroid, macrophage and megakaryocyte (GEMM) lineage and the in vivo chimeric ability. We could see the numbers of 
D
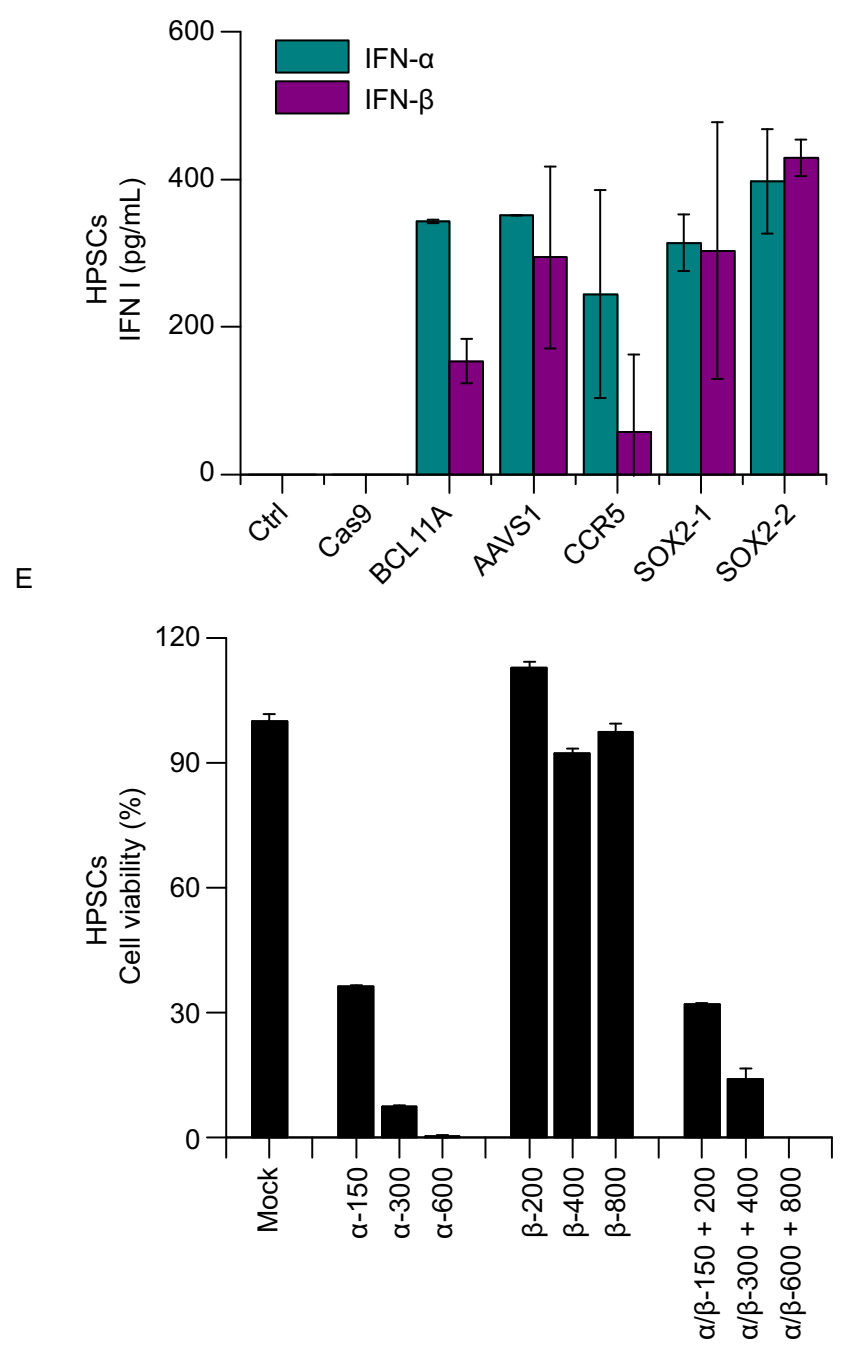

Figure 1. continued.

CFU-GEMM and the chimeric efficiency both improved significantly in CIP treated group contrast to IVT sgRNA group (Figs. 2D, 2E and S6). From above data, we suggested that CIP treatment of IVT sgRNA not only increased cell survival, but also maintained the stemness of HSPCs.

Similar to HSPCs, CIP treatment of IVT RNP (RNP-CIP) targeting various genomic loci also attenuated immunestimulation and improved cell viability of $\mathrm{CD}^{+} \mathrm{T}$ cells and anti-CD19 CAR-T cells (Figs. S7 and S8). These results demonstrated that the 5 ' triphosphate of IVT sgRNA induced type I IFN production, which led to cell death; removing the 5' triphosphate using CIP could rescue these detrimental effects.

We also explored the underlying mechanism of IVT sgRNA induced IFN I release using HeLa cells. We observed the phosphorylation of IRF3 and the expression levels of IFNB1, IFIT1, MDA5 and RIG-I were significantly
$\mathrm{F}$

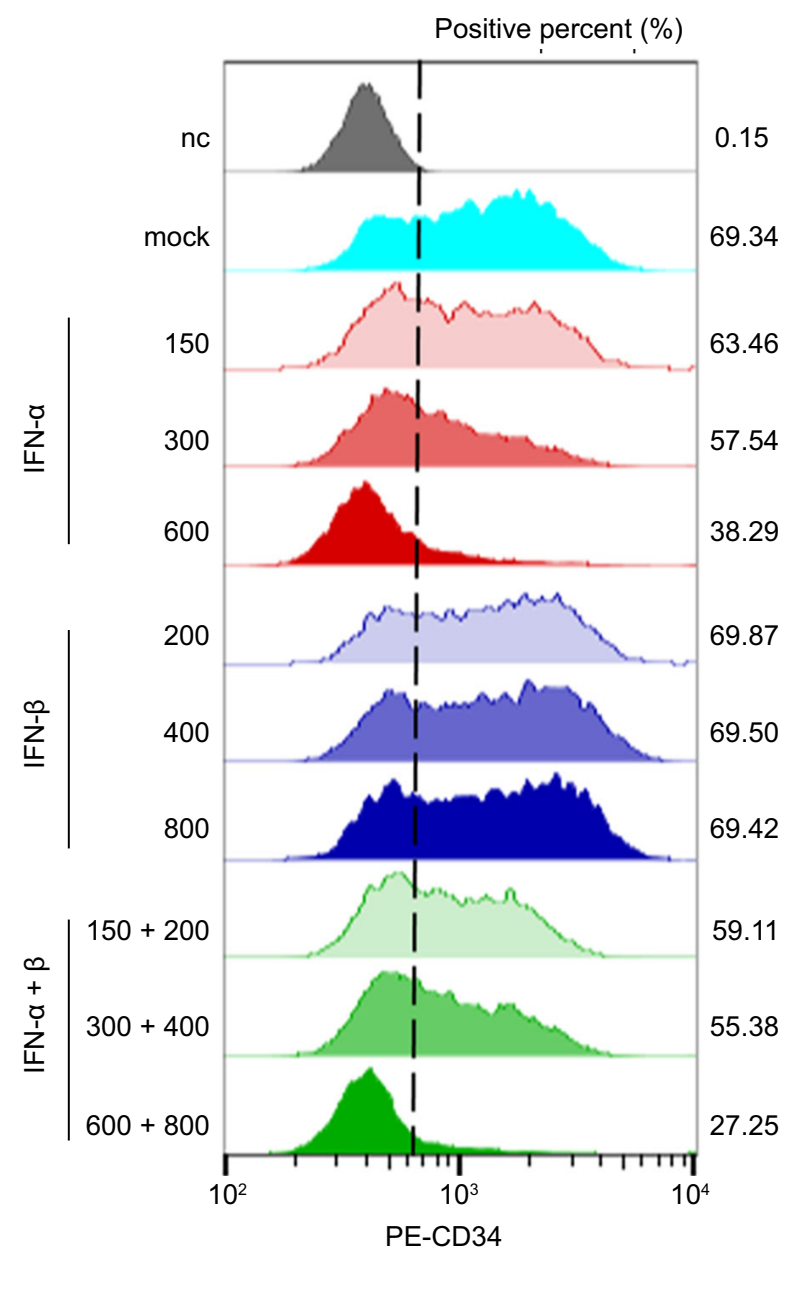

up-regulated in sgRNA-IVT and RNP-IVT electroporation groups (Figs. S9A, S9B, S9C and S10), confirming the innate immunity was also activated in HeLa cells.

Next, we established MDA5 and RIG-I knockout HeLa cell lines (Fig. S9D). Compared with wild type (WT), the expression of IFNB1 and IFIT1 in MDA5 KO was markedly decreased, and was comparable to mock, RIG-I KO and MDA5/RIG-I DKO clones after IVT sgRNA electroporation (Fig. S9E). These results suggested that MDA5 and RIG-I recognized the IVT sgRNA and induced IFN expression.

We next assessed the effect of removing 5' triphosphate of IVT sgRNA via CIP on gene editing efficiency. Firstly, we found that the removal of 5 ' triphosphate did not significantly affect the persistence of sgRNA in 293T cells (Fig. S11A). We further analyzed the gene editing efficiency of sgRNACIP, and found that CIP treatment did not affect the gene editing efficiency of the CRISPR-Cas9 system in HSPCs, T 
D

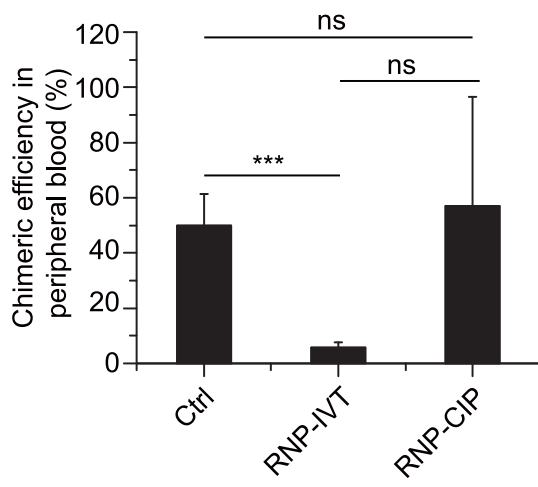

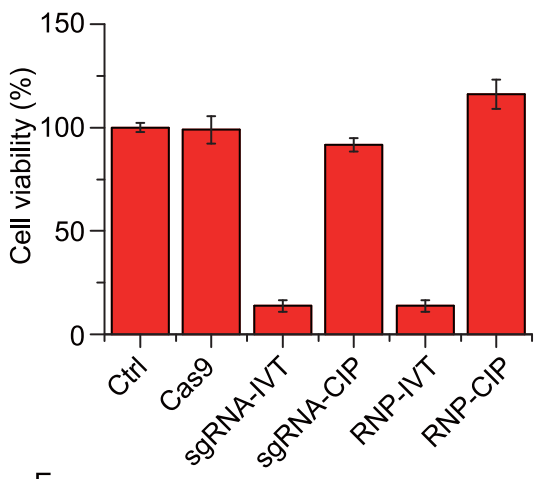

E

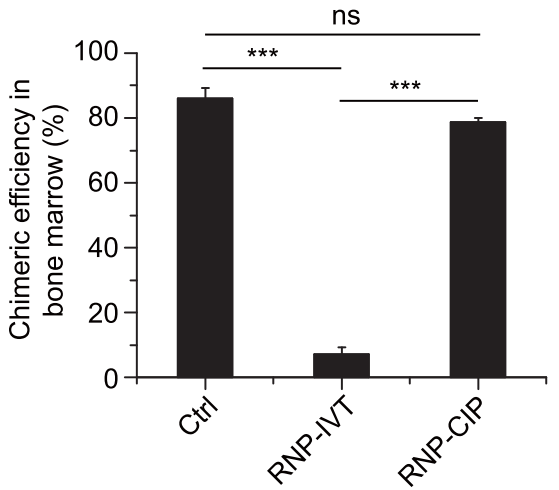

C
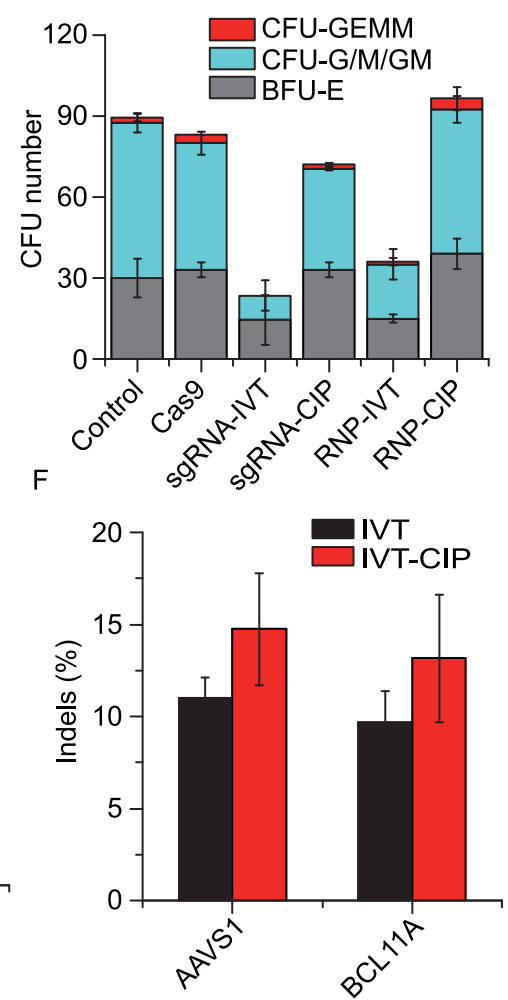

Figure 2. Removing 5' triphosphate of IVT sgRNA by CIP completely rescued the detrimental effects in HSPCs. (A) CIP treatment reduced the production of IFN- $\alpha$ production in HSPCs. (B and C) The cell viability $(B)$ and colony formation ability (C) of HSPCs with CIP treatment were comparable with electroporation mock control. Experiments were performed in cells from two donors, error bars represent SDs for two biological replicates. (D and E) The chimeric efficiencies of HSPCs in NPG mice peripheral blood (D) and bone marrow (E). The chimeric efficiencies were collected 12 weeks post injection. Error bars represent SD for two biological replicates. (F) CIP treatment did not affect the gene editing efficiency of CRISPR-Cas9 system in HSPCs. Indel frequencies were measured by TIDE and bars represent average indel frequencies $\pm S D, n=3$. Ctrl: control; RNP-CIP: CIP treated BCL11A IVT sgRNA + Cas9 protein; RNP-IVT: BCL11A IVT sgRNA + Cas9 protein. $P$ values were calculated by employing unpaired $t$-test comparing with indicated groups. ${ }^{* * *} P<0.001$.

cells, CAR-T cells and various cell lines (Figs. S11B, S11C and S12). Furthermore, performing multiplex gene editing in anti-CD19 CAR-T cells using sgRNA -CIP did not affect the function of CAR-T cells (Fig. S13).

In this study, we showed that the triphosphate at 5' end of IVT sgRNA triggered the innate immunity through R/G-II MDA5 signaling pathway and induced the production of type I IFN, which led to the death of CD34 ${ }^{+}$HSPCs and $\mathrm{CD}^{+}{ }^{\mathrm{T}}$ cells, and stemness reduction in CD34+ HSPCs. By treating the IVT sgRNA with CIP, we were able to eliminate the IFN induction effect of IVT sgRNA, and achieved significantly better cell survival without affecting gene editing efficiency of the CRISPR-Cas9 system. While our manuscript is under review, another group published similar findings in HeLa and T cells, and identified RIG-I as the sensor for IVT sgRNA (Kim et al., 2018). In addition to T and CAR-T cells, our work demonstrated a dramatic effect on both the survival and stemness of human HSPCs, and identified MDA5 as another player involved in innate immunity response induced by IVT sgRNAs.

Although sgRNA generated by chemical synthesis is an option, the generally high costs and long production time limit their widespread application. Moreover, the length of sgRNA is almost reaching the current limits of RNA chemical synthesis. With various optimization and added structures and sequences, sgRNAs longer than $100 \mathrm{nt}$ are commonly used (Shechner et al., 2015; Cheng et al., 2016) and are most conveniently generated by IVT. Therefore, RNA-CIP is useful for a variety of applications in gene editing and transcription regulation of primary cells.

\section{FOOTNOTES}

We would like to thank Junning Wei and Yi Yang (Beijing Cord Blood Bank) for their help in preparing the cord blood samples. We would also like to thank Lei Shi (Beijing Cord Blood Bank) for her help with 
CFU analysis. This work was supported by the Strategic Priority Research Program of the Chinese Academy of Sciences (XDA16010205), National Key Research and Development Program of China (2018YFA0107703), and the National Natural Science Foundation of China (Grant Nos. 81773269 and 31722036). HW is supported by the "Young Thousand Talent Project".

$\mathrm{H}$. Wang conceived and supervised the entire project. W. Mu performed experiments in T and CAR-T cells. N.T. and C.C. performed electroporation of HSPCs and prepared cells for colony formation assays. X. Wei provided human umbilical cord blood. W. Mu, N. Tang, W. Sun, and H. Wang analyzed the data and wrote the manuscript.

Wei Mu, Na Tang, Chen Cheng, Wen Sun, Xiaofei Wei and Haoyi Wang declare that they have no conflict of interest. All procedures followed were in accordance with the ethical standards of the responsible committee on human experimentation (institutional and national) and with the Helsinki Declaration of 1975, as revised in 2000 (5). All donors of umbilical cord blood (UCB) units have provided informed consent. All institutional and national guidelines for the care and use of laboratory animals were followed.

Wei Mu ${ }^{1,2}, \mathrm{Na}$ Tang $^{1,2}$, Chen Cheng ${ }^{1,3}$, Wen Sun ${ }^{1}$, Xiaofei Wei ${ }^{4}$, Haoyi Wang ${ }^{1,2,5 \bowtie}$

1 State Key Laboratory of Stem Cell and Reproductive Biology, Institute of Zoology, Chinese Academy of Sciences, Beijing 100101, China

2 University of Chinese Academy of Sciences, Beijing 100049, China

${ }^{3}$ School of Life Sciences, University of Science and Technology of China, Hefei 230026, China

${ }^{4}$ Beijing Cord Blood Bank, Beijing 100176, China

${ }^{5}$ Institute for Stem Cell and Regeneration, Chinese Academy of Sciences, Beijing 100101, China

$\bowtie$ Correspondence: wanghaoyi@ioz.ac.cn (H. Wang)

\section{OPEN ACCESS}

This article is distributed under the terms of the Creative Commons Attribution 4.0 International License (http://creativecommons.org/ licenses/by/4.0/), which permits unrestricted use, distribution, and reproduction in any medium, provided you give appropriate credit to the original author(s) and the source, provide a link to the Creative Commons license, and indicate if changes were made.

\section{REFERENCES}

Cheng AW, Jillette $N$, Lee P, Plaskon D, Fujiwara Y, Wang W, Taghbalout A, Wang H (2016) Casilio: a versatile CRISPR-Cas9Pumilio hybrid for gene regulation and genomic labeling. Cell Res 26:254-257

Cong L, Ran FA, Cox D, Lin S, Barretto R, Habib N, Hsu PD, Wu X, Jiang W, Marraffini LA et al (2013) Multiplex genome engineering using CRISPR/Cas systems. Science 339:819-823

Dever DP, Bak RO, Reinisch A, Camarena J, Washington G, Nicolas CE, Pavel-Dinu M, Saxena N, Wilkens AB, Mantri S et al (2016) CRISPR/Cas9 beta-globin gene targeting in human hematopoietic stem cells. Nature 539:384-389

Dong-Ho K, Longo M, Young H, Lundberg P, Cantin E, Rossi JJ (2004) Interferon induction by siRNAs and ssRNAs synthesized by phage polymerase. Nat Biotechnol 22:321

Gundry MC, Brunetti L, Lin A, Mayle AE, Kitano A, Wagner D, Hsu JI, Hoegenauer KA, Rooney C, Goodell MA et al (2016) Highly efficient genome editing of murine and human hematopoietic progenitor cells by CRISPR/Cas9. Cell Rep 17:1453-1461

Hendel A, Bak RO, Clark JT, Kennedy AB, Ryan DE, Roy S, Steinfeld I, Lunstad BD, Kaiser RJ, Wilkens AB et al (2015) Chemically modified guide RNAs enhance CRISPR-Cas genome editing in human primary cells. Nat Biotechnol 33:985

Kim S, Kim D, Cho SW, Kim J, Kim J-S (2014) Highly efficient RNAguided genome editing in human cells via delivery of purified Cas9 ribonucleoproteins. Genome Res 24:1012-1019

Kim S, Koo T, Jee H-G, Cho H-Y, Lee G, Lim D-G, Shin HS, Kim J-S (2018) CRISPR RNAs trigger innate immune responses in human cells. Genome Res 28:367-373

Liu X, Zhang Y, Cheng C, Cheng AW, Zhang X, Li N, Xia C, Wei X, Liu X, Wang H (2016) CRISPR-Cas9-mediated multiplex gene editing in CAR-T cells. Cell Res 27:154

Mali P, Yang L, Esvelt KM, Aach J, Guell M, DiCarlo JE, Norville JE, Church GM (2013) RNA-guided human genome engineering via Cas9. Science 339:823-826

Ran FA, Hsu PD, Wright J, Agarwala V, Scott DA, Zhang F (2013) Genome engineering using the CRISPR-Cas9 system. Nat Protoc 8:2281-2308

Ren J, Zhang X, Liu X, Fang C, Jiang S, June CH, Zhao Y (2017) A versatile system for rapid multiplex genome-edited CAR T cell generation. Oncotarget 8:17002-17011

Schumann K, Lin S, Boyer E, Simeonov DR, Subramaniam M, Gate RE, Haliburton GE, Ye CJ, Bluestone JA, Doudna JA et al (2015) Generation of knock-in primary human T cells using Cas9 ribonucleoproteins. Proc Natl Acad Sci USA 112:10437-10442

Shechner DM, Hacisuleyman E, Younger ST, Rinn JL (2015) Multiplexable, locus-specific targeting of long RNAs with CRISPR-Display. Nat Methods 12:664-670

Zhang Y, Mu W, Wang $\mathrm{H}$ (2017) Gene editing in T cell therapy. J Genet Genomics 44:415-422
Wei Mu, Na Tang and Chen Cheng have contributed equally to this work.

Electronic supplementary material The online version of this article (https://doi.org/10.1007/s13238-018-0605-9) contains supplementary material, which is available to authorized users. 